\title{
DSK-Zwischenkonferenz am 12. September 2019 in Mainz
}

\section{Entschließung: Digitalisierung der Verwaltung datenschutzkonform und bürgerfreundlich gestalten!}

Die Bundesregierung will die in der Verwaltung geführten Register modernisieren und plant in diesem Zusammenhang einen einfacheren Zugriff auf dort gespeicherte personenbezogene Daten. Nach Auffassung der Konferenz der unabhängigen Datenschutzaufsichtsbehörden des Bundes und der Länder (Datenschutzkonferenz) darf dieses Vorhaben nicht zur Einführung von einheitlichen, verwaltungsübergreifenden Personenkennzeichen bzw. Identifikatoren führen. Vielmehr muss der Schutz der Grundrechte und Grundfreiheiten, insbesondere das Recht auf Schutz personenbezogener Daten, Priorität haben. Ebenso wichtig ist es, den Bürgerinnen und Bürgern die besseren Dienstleistungen verbunden mit einer deutlich höheren Transparenz anzubieten.

\section{Bundesregierung nimmt Modernisierung der Register in Angriff}

Die Bundesregierung hat mit dem Onlinezugangsgesetz ein umfangreiches Digitalisierungsprogramm für die Verwaltung in Deutschland gestartet. Bund und Länder sind verpflichtet, ihre Verwaltungsleistungen künftig auch elektronisch über Verwaltungsportale anzubieten. Es sollen Nutzerkonten bereitgestellt werden, über die sich Nutzende für die im Portalverbund verfügbaren elektronischen Verwaltungsleistungen von Bund und Ländern einheitlich identifizieren können.

In diesem Zusammenhang hat sich der Nationale Normenkontrollrat (NKR) für eine Modernisierung der deutschen Registerlandschaft ausgesprochen und empfohlen, dass bestimmte Basisdaten von Bürgern und Unternehmen nur einmal mitgeteilt werden müssen („Once Only“-Prinzip). Der NKR hat darüber hinaus angeregt, datenschutzkonforme Identifikationsnummern für Personen, Unternehmen sowie Gebäude, Wohnungen und Flurstücke zu schaffen und zu nutzen und ein „Datencockpit“ einzurichten, bei dem die Bürgerinnen und Bürger alle staatlichen Datenflüsse im Auge haben können.

Die Einführung solcher Identifikationsnummern für Personen wird aktuell unter Federführung des Bundesministeriums des Innern, für Bau und Heimat (BMI) von der Bundesregierung verfolgt. Der IT-Planungsrat hat in seiner 28. Sitzung am 12. März 2019 den vom BMI vorgelegten „Leitlinien für eine Modernisierung der Registerlandschaft“ zugestimmt sowie den „Vorschlag für die Verbesserung des Identitätsmanagements als Teil der Registermodernisierung“ zur Kenntnis genommen und das angestrebte Vorhaben begrüßt.

\author{
Datenschutzfreundliche und transparente \\ Gestaltung für Bürgerinnen und Bürger
}

Insbesondere im Hinblick auf die geplante Verwendung modernisierter Register für zukünftige Zensus-Erhebungen und geplante/modernisierte Zugriffsrechte der Sicherheitsbehörden bedarf es eines besonderen Schutzes der betroffenen Personen. Den hohen Risiken für das Recht auf informationelle Selbstbestimmung muss in einem umfassenden regulatorischen, vor allem aber technischen und organisatorischen Konzept begegnet werden. Nur so können die vom deutschen und europäischen Verfassungsrecht geforderten Garantien gewahrt werden.

Die Modernisierung der Register muss zwingend von Beginn an auch dafür genutzt werden, den Bürgerinnen und Bürgern die Nutzung der im Online-Zugangsgesetz vorgesehenen Dienstleistungen durch Nutzung einmal hinterlegter Daten zu erleichtern. Von besonderer Bedeutung ist es darüber hinaus, den Bürgerinnen und Bürgern ein im Vergleich zur gegenwärtigen Situation deutlich höheres Maß an Transparenz zu gewährleisten. Ein „Datencockpit“, wie es der NKR bereits vorgeschlagen hat, muss es den Bürgerinnen und Bürgern erlauben, jederzeit nachzuvollziehen, welches Register welche Daten über sie vorhält, welche Behörden darauf zugegriffen haben und mit welchen anderen Daten diese verknüpft wurden. Gleichzeitig muss gewährleistet sein, dass ausschließlich den betroffenen Bürgerinnen und Bürgern der Zugriff möglich ist. Auf dieser Grundlage muss die Digitalisierung der Verwaltung dazu genutzt werden, das informationelle Machtgefälle zwischen Staat und Bürgerinnen und Bürgern weitgehend aufzuheben und ihnen die Inanspruchnahme ihrer Rechte deutlich zu erleichtern.

Dazu muss nach Auffassung der Datenschutzkonferenz die dezentrale Registerstruktur erhalten bleiben. Die Nutzung von einheitlichen, verwaltungsübergreifenden Personenkennzeichen bzw. Identifikatoren zur direkten Identifizierung von Bürgerinnen und Bürgern lehnt die Datenschutzkonferenz ab. Sie fordert alternative Methoden zur eindeutigen Identifizierung. Neben Abgleichen über den jeweiligen Datensatz des Registers kämen dafür allenfalls sektorspezifische Personenkennziffern in Betracht, die eine eindeutige Identifizierung erlauben, einseitigen staatlichen Abgleich von Daten verhindern, ein Höchstmaß an Transparenz beispielsweise durch ein Datencockpit ermöglichen, das Risiko von Missbrauch und Kompromittierung verringern und die Eindeutigkeit von Registern gewährleisten. 